\title{
Raman Piezospectroscopy of Phonons in Bulk 6H-SiC
}

\author{
K. Grodecki ${ }^{a, b}$, A. Wysmolek ${ }^{a}$, R. Stępniewski ${ }^{a}$, J.M. Baranowski $^{a, b}$, W. Hofman $^{b}$, \\ E. TYMICKI ${ }^{b}$ AND K. GRASZA ${ }^{b, c}$ \\ ${ }^{a}$ Institute of Experimental Physics, Faculty of Physics, University of Warsaw, Hoża 69, 00-681 Warsaw, Poland \\ ${ }^{b}$ Institute of Electronic Materials Technology, Wólczyńska 133, 01-919 Warsaw, Poland \\ ${ }^{c}$ Institute of Physics, Polish Academy of Sciences, al. Lotników 32/46, 02-668 Warsaw, Poland
}

\begin{abstract}
Raman piezospectroscopy of high quality $6 \mathrm{H}-\mathrm{SiC}$ crystals is presented. The crystals used in experiments were grown by the seeded physical vapor transport method. Uniaxial stress up to $0.9 \mathrm{MPa}$, obtained using a spring apparatus, was applied along [11-20] and [10-10] directions. It was found that the application of uniaxial stress led to different energy shifts of the observed phonon excitations in the investigated $6 \mathrm{H}-\mathrm{SiC}$ crystals. The obtained pressure coefficients vary in the range $0.98-5.5 \mathrm{~cm}^{-1} \mathrm{GPa}^{-1}$ for different transverse optical phonon modes. For longitudinal optic phonon modes pressure coefficients in the range $1.6-3.6 \mathrm{~cm}^{-1} \mathrm{GPa}^{-1}$ were found. The data obtained could be useful in evaluation of local strain fields in $\mathrm{SiC}$ based structures and devices including epitaxial graphene.
\end{abstract}

PACS numbers: 62.50.-p, 42.65.Dr, 63.20.-e, 78.30.-j

\section{Introduction}

The Raman spectroscopy is one of the classical nondestructive methods to study phonon excitations in semiconductors. This method could be substantially extended when the experiments are performed under uniaxial stress applied along specific crystallographic axes of the investigated crystals. The application of the Raman piezospectroscopy to silicon carbide was very limited, since this technique requires high quality, well oriented bulk crystals, free of micropipes, capable to sustain high uniaxial stresses. That is why the knowledge of stress coefficient of phonon excitations in $\mathrm{SiC}$ was restricted to the experiments in which only hydrostatic pressure was used [1, 2]. Recently high quality $6 \mathrm{H}-\mathrm{SiC}$ crystals, which match superior requirements of piezospectroscopic experiments, were grown at the Institute of Electronic Materials Technology in Warsaw [3]. This allowed us to carry out Raman scattering experiments on $6 \mathrm{H}-\mathrm{SiC}$ crystals under uniaxial stress presented here. The results provide information about stress coefficients in $\mathrm{SiC}$ and can be useful in strain analysis of $\mathrm{SiC}$ based devices.

\section{Experimental details}

The $6 \mathrm{H}-\mathrm{SiC}$ crystals used in the piezospectroscopic experiments were grown by the seeded physical vapor transport (PVT) method on $c$-face (0001) of $6 \mathrm{H}-\mathrm{SiC}$ seeds in argon atmosphere. A graphite resistance heater was used to achieve growth temperature in the range $2100-2300{ }^{\circ} \mathrm{C}$. Two X-ray-oriented $6 \mathrm{H}-\mathrm{SiC}$ samples with the dimensions of $10 \times 1.4 \times 1.6 \mathrm{~mm}^{3}$ were studied.
Uniaxial stress up to $0.9 \mathrm{MPa}$, obtained using a spring apparatus, was applied along $X([11-20])$ and $Y([10-10])$ directions, which are perpendicular to the $c$-axis of the investigated $6 \mathrm{H}-\mathrm{SiC}$ crystals. The Raman scattering experiments were performed at room temperature in backscattering geometry using $532 \mathrm{~nm}$ line from Nd-YAG laser as a source of continuous wave excitation. The scattered light, propagating perpendicularly to the stress direction, was collected in two configurations: with the $k$-vector of light being parallel $(k \| c)$ or perpendicular $(k \perp c)$ to the $c$-axis of the investigated $6 \mathrm{H}$-SiC crystals.

\section{Results and discussion}

Representative Raman spectra of $6 \mathrm{H}-\mathrm{SiC}$ obtained for $X$ and $Y$ oriented samples in the absence of the external uniaxial stress are presented in Fig. 1.

The Raman spectra obtained for both $X$ and $Y$ oriented sample are very similar. They are dominated by a group of peaks due to TO-phonon excitations and LO-phonon mode. The observed excitations can be attributed to folded phonon modes of different symmetry [4]. Two modes of $E_{2}$ symmetry: $\mathrm{TO}(\mathrm{X} 1 / 3)$ and $\mathrm{TO}(\mathrm{X} 1)$ are observed at $784 \mathrm{~cm}^{-1}$ and $812 \mathrm{~cm}^{-1}$, respectively. The $\mathrm{LO}(\mathrm{X} 0)$ phonon mode of $A_{1}$ symmetry is present at $978 \mathrm{~cm}^{-1}$. It is observed that the spectra measured for $k \| c$ and $k \perp c$ configurations in the absence of the external uniaxial stress show different intensity patterns. This reflects the properties of selection rules obeyed in the investigated system.

In Fig. 2 the Raman spectrum obtained for $k \perp c$ configuration for $\mathrm{TO}(\mathrm{X} 1)$ mode under applied pressure of 


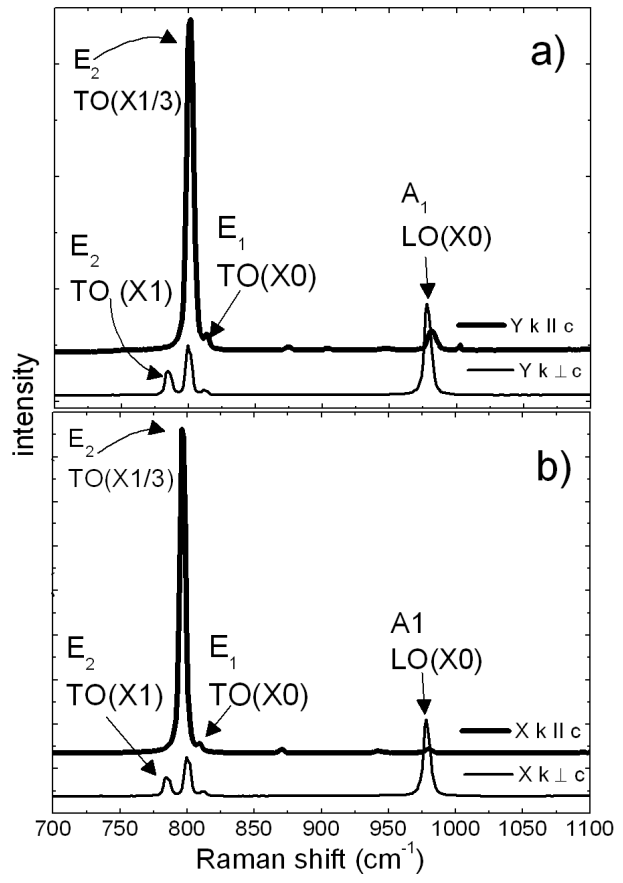

Fig. 1. Raman spectra of $6 \mathrm{H}-\mathrm{SiC}$ samples oriented along (a) $Y$ [11-20] and (b) $X$ [10-10] directions for $k \| c$ and $k \| c$ light propagation.

$0.9 \mathrm{MPa}$ along $Y$ direction is presented, together with zero-stress spectrum. In spite of the fact that the observed shift is rather small, the central position of the peak can be evaluated for each applied stress value. In Fig. 3 stress dependences obtained for $\mathrm{TO}(\mathrm{X} 1 / 3)$ as well as for $\mathrm{LO}(\mathrm{X} 0)$ phonon modes are shown.

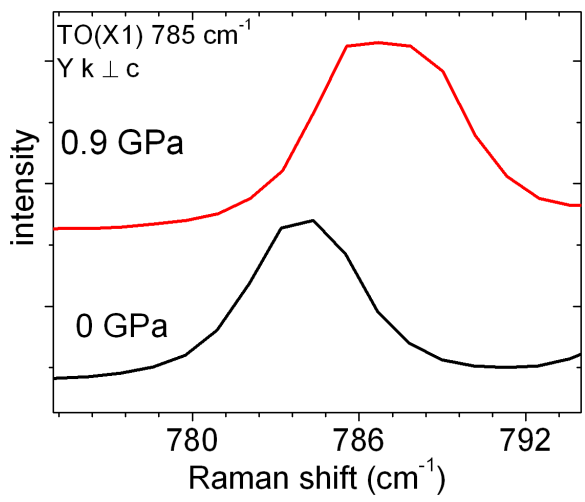

Fig. 2. The Raman spectra due to the TO(X1) phonon mode obtained for $k \perp c$ configuration at $0.9 \mathrm{GPa}$ (upper curve) and in the absence of external stress (lower curve).

It is observed that under external stress the modes show linear energy shifts with pressure coefficients dependent both on the stress direction and the light propagation direction (Fig. 3). This is a manifestation of the symmetry of particular phonon excitations in $6 \mathrm{H}-\mathrm{SiC}$.

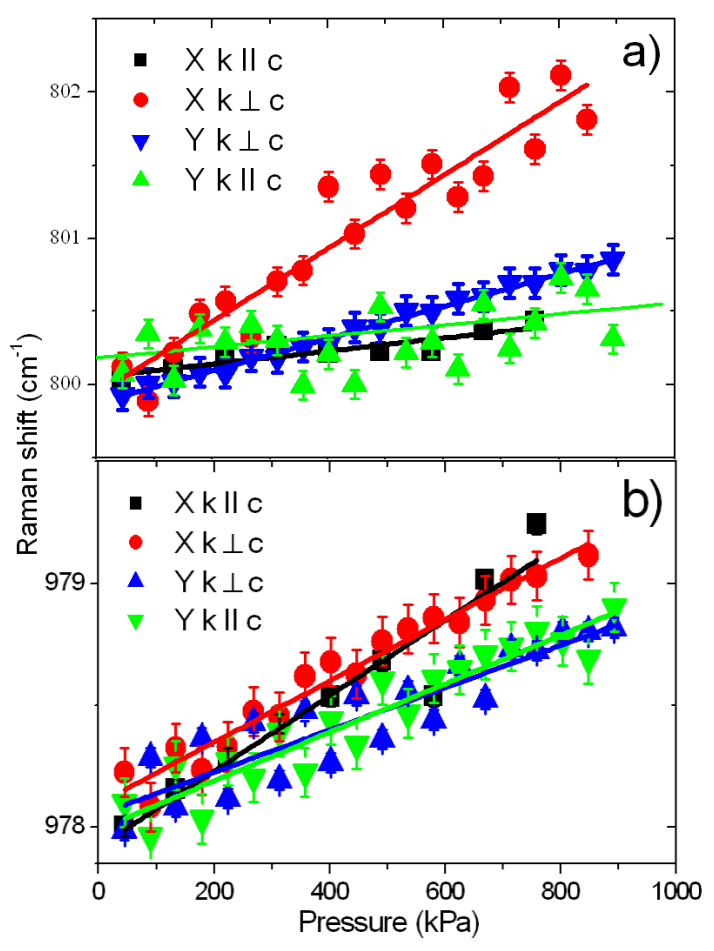

Fig. 3. Pressure dependence of Raman excitations obtained for $\mathrm{TO}(\mathrm{X} 1 / 3)$ (a) and $\mathrm{LO}(\mathrm{X} 0)$ (b) phonon modes for different stress and light propagation directions.

The pressure coefficients obtained for different stress and light propagation directions are collected in Table.

TABLE

Pressure coefficients obtained for different phonon modes under uniaxial stress applied along $X$ and $Y$ direction for different orientation of the light $k$-vector with respect to the $c$-axis of the $6 \mathrm{H}$-crystals.

\begin{tabular}{c|c|c|c|c|c}
\hline \hline $\begin{array}{l}\text { Stress } \\
\text { direction }\end{array}$ & $\begin{array}{l}k \text {-vector } \\
\text { direction }\end{array}$ & $\begin{array}{l}E 2 \\
\mathrm{TO}(\mathrm{X} 1)\end{array}$ & $\begin{array}{l}E 2 \\
\mathrm{TO}(\mathrm{X} 1 / 3)\end{array}$ & $\begin{array}{l}E 1 \\
\mathrm{TO}(\mathrm{X} 0)\end{array}$ & $\begin{array}{l}A 1 \\
\mathrm{LO}(\mathrm{X} 0)\end{array}$ \\
\hline & & \multicolumn{4}{|c}{$\left[\mathrm{cm}^{-1} \mathrm{GPa}^{-1}\right]$} \\
\hline$X$ & $k \| c$ & & 0.44 & & 1.55 \\
$X$ & $k \perp c$ & 2.58 & 2.16 & 2.35 & 1.26 \\
$Y$ & $k \perp c$ & 1.13 & 1.10 & 1.31 & 0.87 \\
$Y$ & $k \| c$ & & 0.38 & & 0.99
\end{tabular}

The obtained uniaxial stress coefficients of different phonon modes are smaller than the values reported for the hydrostatic pressure, which are of about $3.8 \mathrm{~cm}^{-1} \mathrm{GPa}^{-1}$ for $\mathrm{LO}$ phonon mode and $3.1 \mathrm{~cm}^{-1} \mathrm{GPa}^{-1}$ for TO phonon mode $[1,2]$. The obtained data would provide information about stress coefficients in $\mathrm{SiC}$ in the way proposed in Ref. [5]. The obtained information could also be useful for the analysis of strain effects in the SiC-based structures and devices, especially in the case of graphene structures grown by annealing of $\mathrm{SiC}$ at high temperatures [6]. Since graphene 
layers are usually grown on [0001]-oriented substrates, backscattering Raman experiments would easily provide information about strain components perpendicular to the $c$-axis, thus corresponding to the $k \| c$ configuration examined in this paper. Performing Raman experiments on epitaxial graphene layers, simultaneous information on phonon excitations in $\mathrm{SiC}$ could be obtained. This would allow for an evaluation of the influence of strain fields in the $\mathrm{SiC}$ substrate on the optical and electrical properties of graphene layers studied [7-9].

\section{Conclusions}

The Raman experiments in high quality $6 \mathrm{H}-\mathrm{SiC}$ crystals were performed. Preliminary data show that application of uniaxial stress (up to $0.9 \mathrm{MPa}$ ) leads to different linear energy shifts of the phonon excitations in the $6 \mathrm{H}-\mathrm{SiC}$ crystals. The results obtained allow to determine stress coefficients in $\mathrm{SiC}$ and can be useful in evaluation of strain effects in the SiC-based structures.

\section{Acknowledgments}

The work was partially supported by Polish Ministry of Science and Higher Education, Grants PBZ-
-MEiN-6/2/2006 and DWM/N179/PICS-FR/2008 and EU project No. MTKD-CT-2005-029671.

\section{References}

[1] A. Debernardi, C. Ulrich, K. Syassen, M. Cardona, Phys. Rev. B 59, 10 (1999).

[2] C. Ulrich, A. Debernardi, E. Anastassakis, K. Syassen, M. Cardona, Phys. Status Solidi B 211, 293 (1999).

[3] A. Wysmołek, K. Wardak, R. Stepniewski, J.M. Baranowski, M. Potemski, E. Tymicki, K. Grasza, Acta Phys. Pol. A 110, 437 (2006).

[4] S. Nakashima, H. Harima, T. Tomita, T. Suemoto, Phys. Rev. B 62, 24 (2000).

[5] Z.C. Feng, W.J. Choyke, J.A. Poweil, J. Appl. Phys. 64, 12 (1988).

[6] J. Borysiuk, R. Bożek, W. Strupiński, A. Wysmołek, K. Grodecki, R. Stępniewski, J.M. Baranowski, J. Appl. Phys. 105, 023503 (2009).

[7] Z.H. Ni, T. Yu, Y.H. Lu, Y.Y. Wang, Y.P. Feng, Z.X. Shen, Am. Chem. Soc. Nano 2, 2301 (2008).

[8] N. Ferralis, R. Maboudian, C. Carraro, Phys. Rev. Lett. 101, 156801 (2008).

[9] J.A. Robinson, C.P. Puls, N.E. Staley, J.P. Stitt, M.A. Fanton, K.V. Emtsev, T. Seyller, Y. Liu, Nano Lett. 9, 3 (2009). 